\title{
Conservation of the Lacquer Artifacts Excavated from Suchon-ri, Gongju, Korea
}

\author{
Ji Ae Song' | Ah-reum Jeong* | Hyeok-nam Kwon** | Woo-rim Han*** | Hyun-sang Lee ${ }^{* * * *}$ \\ Cultural Heritage Conservation Science Center, National Research Institute of Cultural Heritage, \\ Daejeon, 34122, Korea \\ *Presidential Archives, National Archives of Korea, Sejong, 30107, Korea \\ **Conservation Science Division, National Research Institute of Cultural Heritage, Daejeon, 34122, Korea \\ ***Gaya National Research Institute of Cultural Heritage, Changwon, 51430, Korea \\ $* * * *$ Chungnam Institute of History and Culture, Gongju, 32589, Korea \\ ${ }^{1}$ Corresponding Author: ssong@korea.kr, +82-42-860-9383
}

\begin{abstract}
In November 2011, various artifacts were excavated from the No. 8 stone-lined tomb in Suchon-ri, Gongju by the Chungnam Institute of History and Culture. These included artifacts with lacquered mainframe and silvery metal ornament. These were recovered together with soil and underwent conservation treatment.

In this paper, we discuss the scientific analysis and conservation treatment of the lacquered artifacts excavated from the Suchon-ri site. Among our findings is that the artifacts have three layers of lacquer coating and the metal parts are primarily composed of Ag. As the artifacts were recovered with soil from the site, the soil was removed from underneath and inside the artifacts. The inside of the lacquerware was reinforced with rayon paper using $3 \%$ funori, and the metal was treated with acrylic resin after removing the acrylic resin previously used to recover the artifact, followed by cleaning. The metal was also internally reinforced with gauze. Finally, the interior reinforcement was made using PVA and resin in pulp and attached with funori to preserve the shape of the lacquerware, and the exposed rayon was finished with acrylic paint. The main advantages of this study are its review of conservation treatment strategies for lacquer artifacts whose numbers have recently increased, and the application of new conservation treatment methods.
\end{abstract}

Key Words: Gongju, Suchon-ri, Lacquerware, Conservation, Sliver

\section{INTRODUCTION}

The Suchon-ri tomb Group I is located at Suchon-ri 221-3, Euidang-myeon, Gongju-si, and various remains and artifacts dating from the Bronze Age to the Joseon Dynasty were excavated in a cultural historic site excavation. Various major artifacts including a golden crown, goldbronze shoes, Chinese porcelain, and gold-bronze monument were excavated from six remains of the Baekje period found at Site II, and designated as National Historic Site No. 460 on March 3, 2005. From the excavation of the lower part of the Suchon-ri tombs group(the first excavation area in 2011)(Figure 1), 25 remains were identified: one Bronze Age settlement, six pit-style remains, ten pit-style remains of the Proto-Three Kingdom period, two stonelined tombs of the Baekje period, one stone chamber tomb, one pit tomb, one pit-style remains, one stone-lined tomb of unknown era, and two pit-style remains (Chungnam Institute of History and Culture, 2013)(Figure 2).

Among the remains, No.8 stone-lined tomb of Baekje period is a rectangular burial located approximately $6 \mathrm{~m}$ southwest of No.9 pit burial of Paekje Period and No.7 stone-lined tomb of Baekje period. The No.8 stone-lined tomb is an underground pit with a lining made of trimmed stone. Its long axis is toward $30^{\circ}$ west from the north-south direction and arranged to be parallel to the contour line. The planar form is a horseshoe-shaped square with a length of $600 \mathrm{~cm}$, a width of $389 \mathrm{~cm}$, and a depth of $160 \mathrm{~cm}$, and 
many artifacts were excavated from it. In the tomb, a wooden coffin was found with a lacquered artifacts, gold-bronze shoes, and gold earrings among others in the midsection in the northeast side(Chungnam Institute of History and Culture, 2013)(Figure 2). The lacquer artifacts was black and its cross section was close to an elliptical shape. Its head was without its top, and the lower end of the handle was finished in a metal hemisphere. The excavating organization concluded that the artifacts was a handle of a fan based on the state of the artifacts.

In this paper, we discuss scientific analysis and conservation treatment of the lacquered artifacts excavated from the Suchon-ri, Gongju. Although studies on conservation treatment of lacquered artifacts(Lee and Han, 2017; Lim and Fumio, 2018) exist, conservation cases of threedimensional lacquered artifacts, especially those with lacquer layers only with a loss of the object under the layers, are rare; therefore, we discuss practical issues that emerged during conservation treatment of the artifacts in

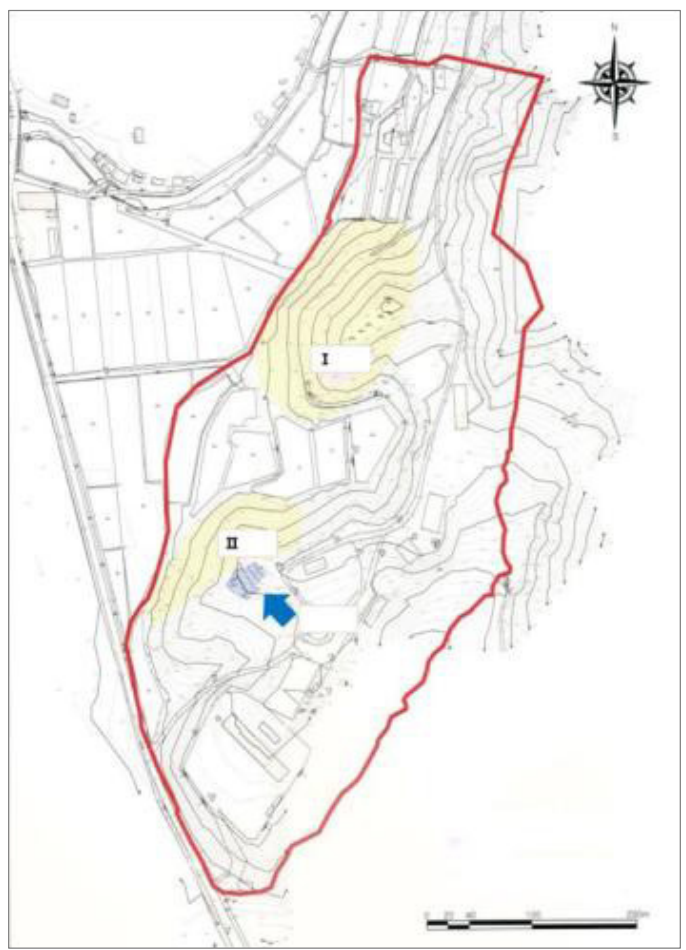

Figure 1. Face map of Suchon-ri tombs group excavation area. this paper.

\section{ARTIFACT RECOVERY}

No.8 stone-lined tomb in Suchon-ri, Gongju where the lacquered artifacts was excavated is located at a very low hill of approximately $50 \mathrm{~m}$ above sea level(Figure 3, 7). The lacquered artifacts was excavated from the midsection of the northeast side in a wood coffin. The interior of the artifact was lacquered in black, and the tip was made of metal.

The cross section of the lacquer artifacts was close to an elliptical shape, and its linear head was without its top, and the lower end of the handle was finished in a metal hemisphere(Figure 4). The excavating organization concluded that the artifacts was a handle of a fan based on the state of the artifacts.

The lacquered artifacts was recovered with soil from the site, and the metal part was dehydrated with ethanol and

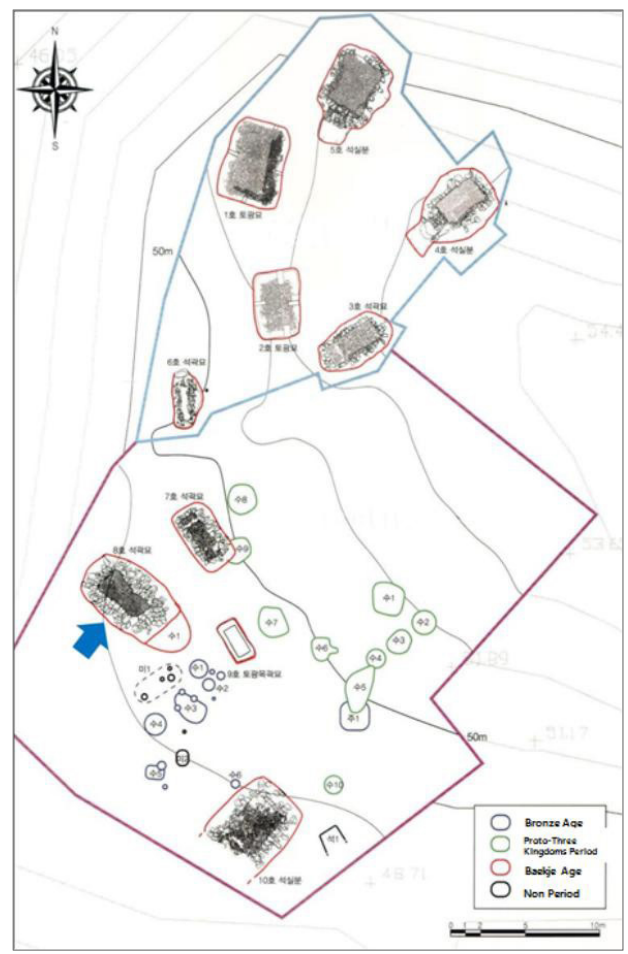

Figure 2. Face map of remains at No.8 stone-line tomb ( $\nearrow:$ No.8 stone-lined tomb) 
cured with acrylic resin(Palaroid B-72) to prevent corrosion. We also watered the lacquer layers to prevent them from drying and curling, and reinforced the surface with Korean paper when recovering the artifacts. The artifacts had a lacquered mainframe and silvery metal ornament(Figure 5,6). The upper part of the artifacts was exfoliating and the metal ornament was caved in the artifacts.

\section{SCIENTIFIC ANALYSIS}

\subsection{Analysis of lacquer}

The detached lacquerware piece from the artifacts was reattached using cold mounting with epoxy resin(Epofix, Struers, USA). The specimen for analysis underwent rock trimming with a cutter with a diamond tip(Minitom,

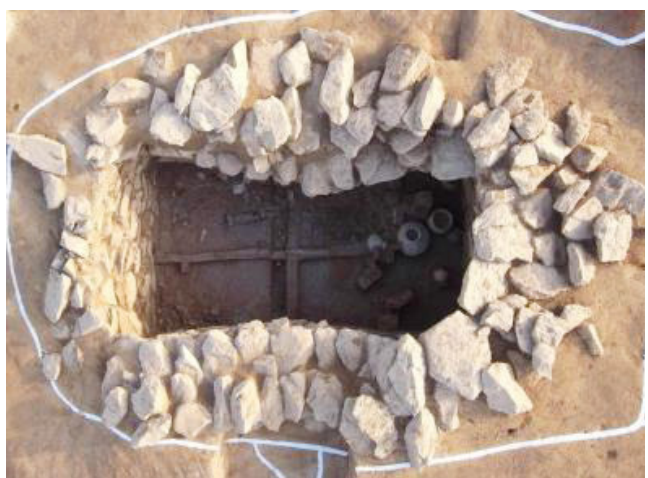

Figure 3. A full view of No.8 stone-lined tomb.

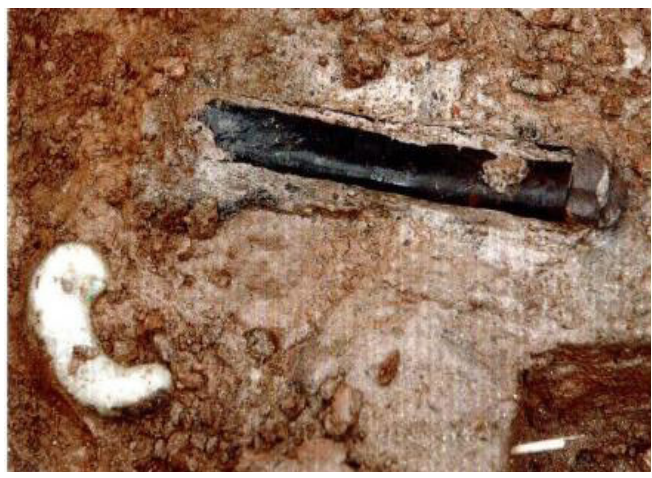

Figure 4. A photo of excavation of lacquered artifacts.
Struers, USA) designed to expose the lacquer piece. We prepared a thin slice using a microtome with a tungsten blade(HM450, Thermo scientific, USA), and examined with a light-transmitting optical microscope(ECLIPS I, Nikon, Japan) and pilarization microscopic(ECLIPS LV100N POL, Nikon, Japan).

The lacquerware piece had scales peeling off starting from the outermost surface due to deterioration, making it difficult to determine the accurate shape of the piece. Despite the difficulty in identification of the piece due to scaling, we were able to identify some of the outermost boundary of layer layers and determined that the piece was composed of at least three lacquer layers(Figure 8). In the polarizing microscope, transparent particles which were not observed in the optical microscope were observed inside the layer(Figure 9).

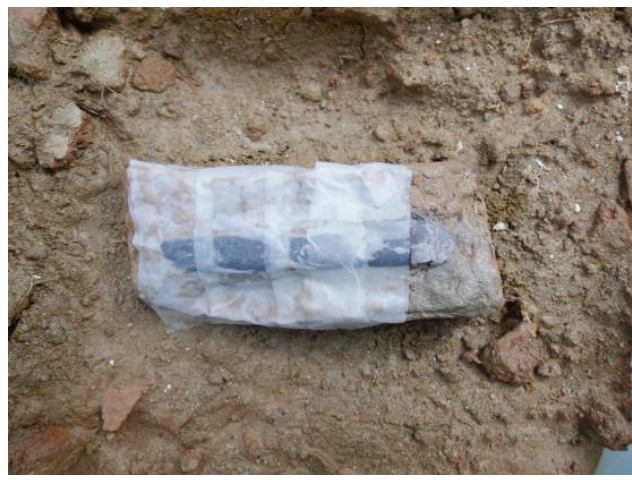

Figure 5. A photo of recovery of lacquered artifacts.

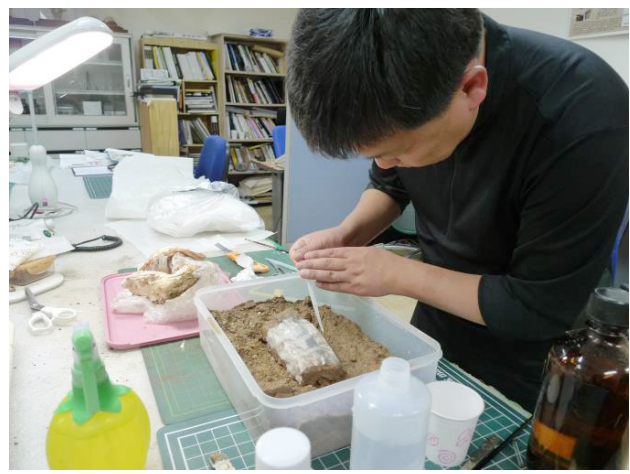

Figure 6. Reinforcing lacquered artifacts with Korean paper. 


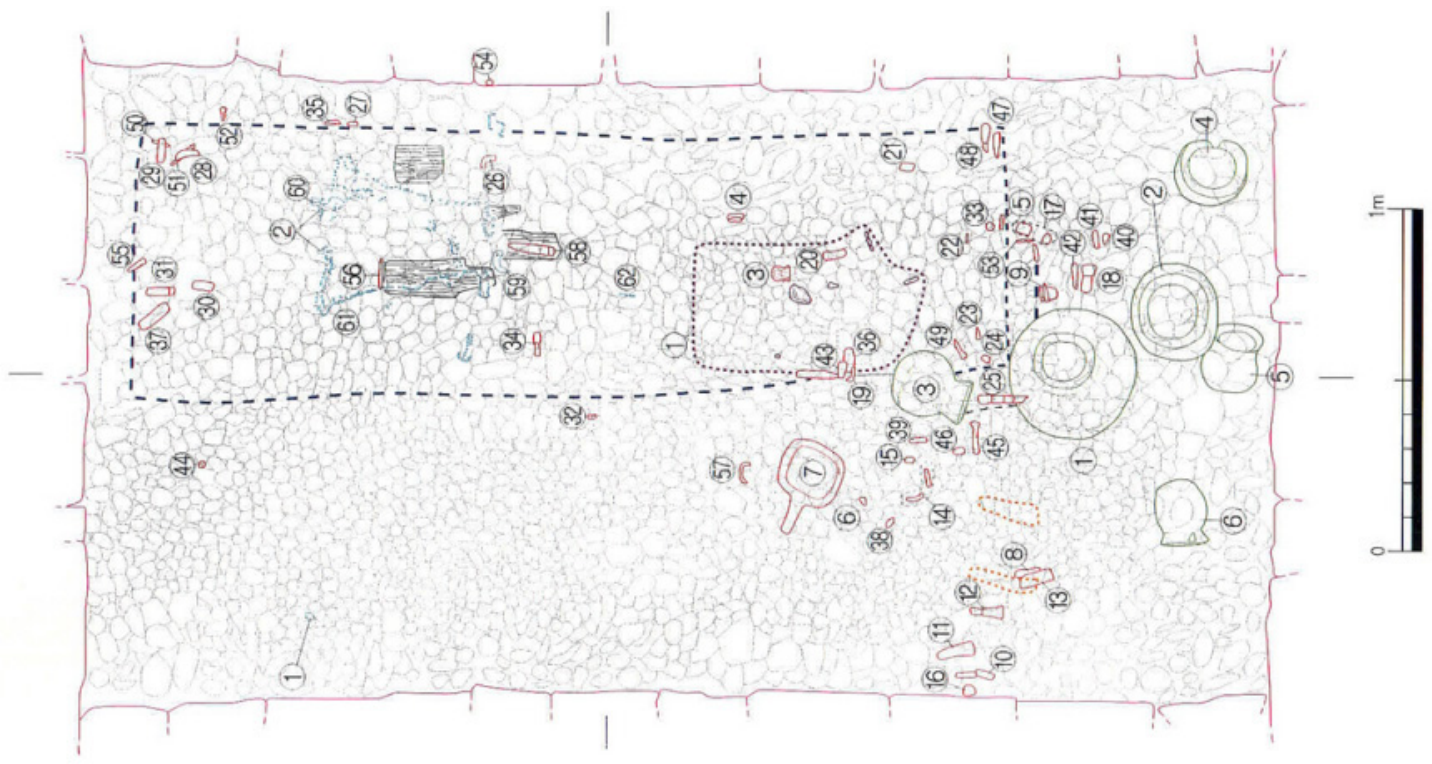

Figure 7. Artifacts excavated from wood coffin(lacquered artifacts-58).

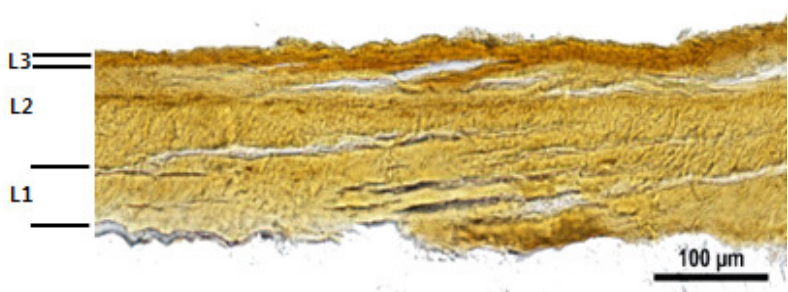

Figure 8. A microscopic photo of lacquerware piece.

\subsection{Analysis of metal}

\subsubsection{XRD}

The component analysis of the metal part was conducted using an X-ray diffractometer(EMPYREAN, PANalytical co., Netherlands) with $\mathrm{Cu}$ as anode material and a $40 \mathrm{kV}, 30$ $\mathrm{mA}$ generator. In the analysis, silver chloride $(\mathrm{AgCl})$ and Silver(Ag) was detected from the metal part(Figure 10).

\subsubsection{Micro-XRF}

The component analysis of the metal part was also conducted using an energy dispersive X-ray fluorescence spectrometer(Eagle 3-XXL, EDAX Inc., USA) for a part of the metal, with a $40 \mathrm{kV}, 500 \mu \mathrm{A}$ generator. The analysis results suggest that the metal is primarily made of $\mathrm{Ag}$. The

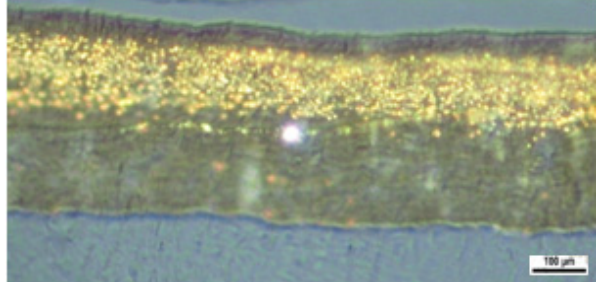

Figure 9. A polarization microscopic photo of lacquerware piece.

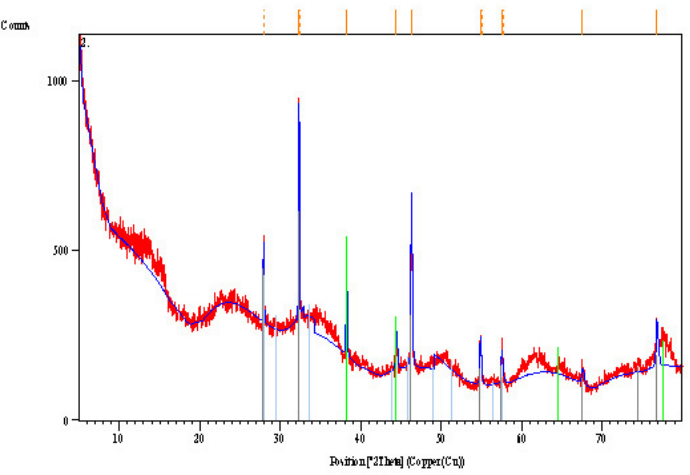

Figure 10. XRD Results of metal part(XRD) (bule : AgCl, green: Ag).

trace amounts of $\mathrm{Mg}, \mathrm{Al}, \mathrm{Si}, \mathrm{P}, \mathrm{Ca}$, and Fe likely to reflect soil components(Figure 11). 

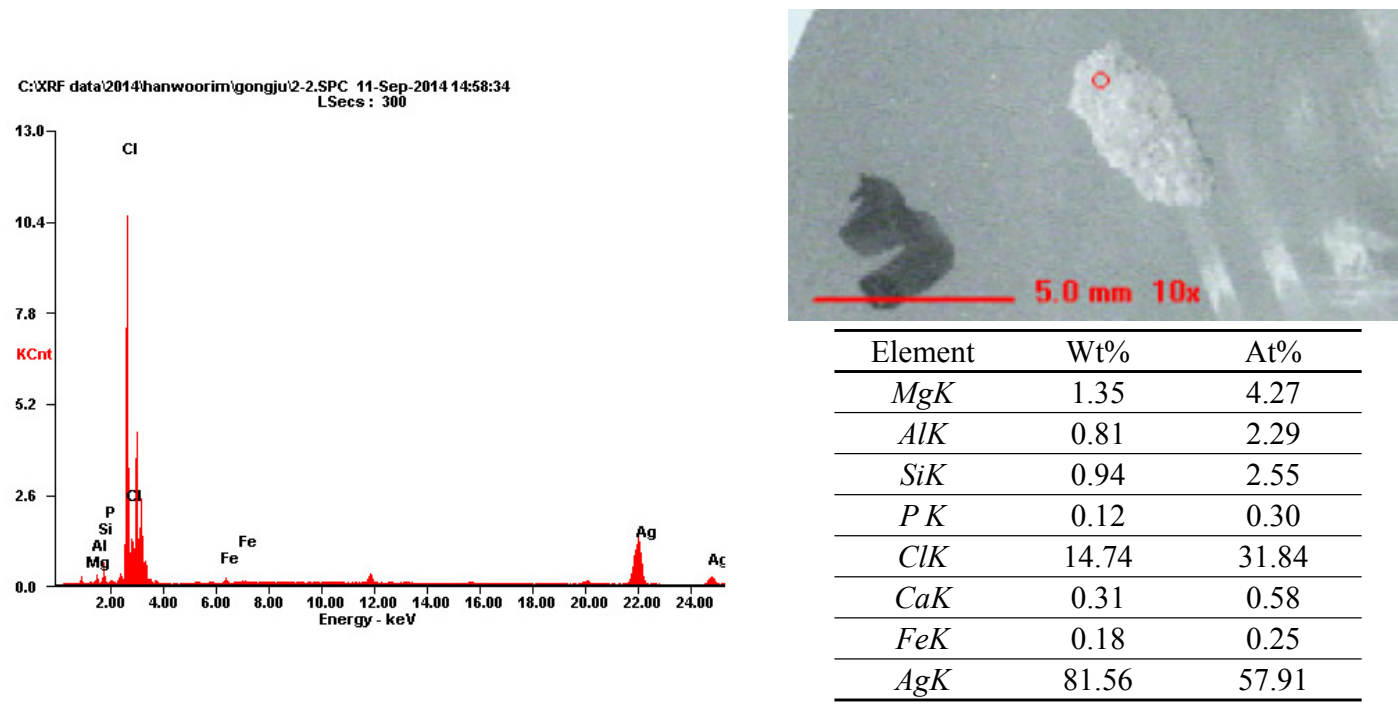

Figure 11. Results of XRF.

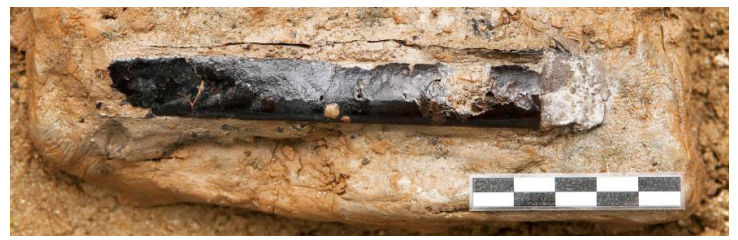

Figure 12. Condition of lacquered artifacts before treatment.

\section{CONSERVATION}

The lacquered artifacts was transferred to the center for full-scale conservation treatment. Before the treatment, the artifacts was photographed and underwent a basic examination.

\subsection{Nondestructive irradiation survey}

The lacquered artifacts had both the parts with lacquer layers and the metal, and the metal part was found in only one side according to an eye inspection. We performed an $\mathrm{X}$-ray analysis using a light X-ray generator(EX-220GH-3, SOFTEX, Japan) to examine the condition and structure of the interior of the lacquer layers. In the analysis, no metal was found in the lacquer part, and the metal part was broken into several depressed pieces(Figure 12, 13).

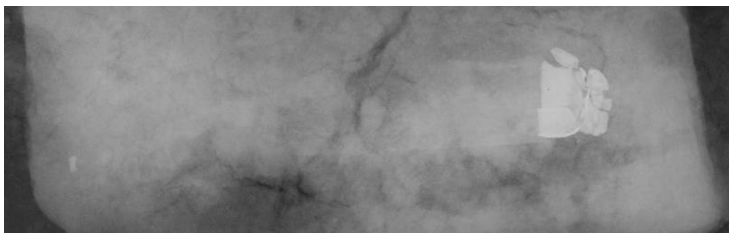

Figure 13. Result of X-ray.

\subsection{Reinforcement of the upper part and soil removal}

The lacquered artifacts was recovered along with adjacent soil at the excavation site after brushing off soil as much as possible. To remove the attached soil, we reinforced the upside of the artifacts after filling the interior cavity and the exterior with soil to prevent the thin layer from breaking due to its load. We used diluted paper paste to reinforce the upper part and applied a plastic film between the lacquer layer and the paper paste to prevent the artifacts from drying and make it easy to remove paper paste later(Figure 14).

After the paper paste was dried completely, we turned the artifacts upside down to examine the bottom side and removed soil at the bottom. We could detect both the lacquer part and the metal part at the bottom side as in the upper part, and found the bottom side in better condition than the upper part despite some cracks in the lacquer 

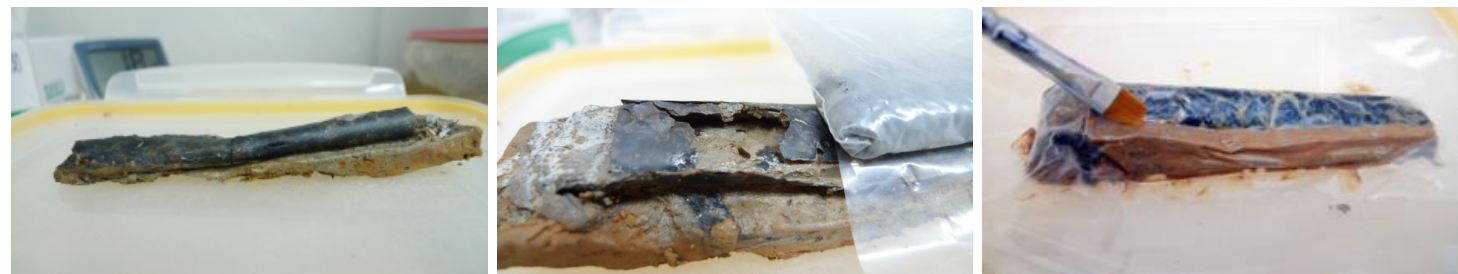

Figure 14. Conservation of facing (Left: soil removal around the artifacts, Center: peeled off layers, Right: facing the upper part).
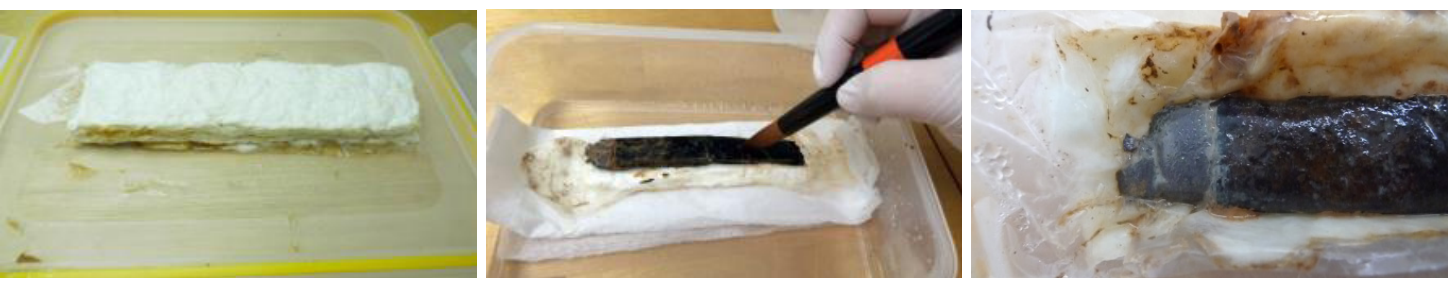

Figure 15. Conservation of support (Left: support frame for the upper part, Center: soil removal from the upper part, Right: exposed metal part at the bottom).

layers. We also reinforced the bottom side after applying a plastic film between lacquer layers and paper paste as in the upper part reinforcement.

After the paper paste was fully dried, we again turned the artifacts upside down to expose the upper part of the artifacts and removed paper paste using distilled water. We also removed soil from the interior cavity while checking if there is any remaining layer. During the soil removal, we found some wooden pieces, but not enough for analysis. We removed soil with soft brushes after softening it with distilled water, or by pouring in distilled water(Figure 15).

\subsection{Reinforcement of the interior with rayon paper}

The lacquered artifactss with soil removed was in a very weak condition although it retained some of its elliptical shape. Therefore, we reinforced the broken pieces due to deterioration and lacquer layers with facing to strengthen them and create a supportive layer. We used rayon paper(12 $\mathrm{g} / \mathrm{m}^{2}$ ) as reinforcement, and experimented with natural and synthetic adhesives including glue, funori, hydroxypropyl cellulose(HPC) and methyl cellulose. Based on the experiment, we chose funori as it did not affect the lacquerware, had high reversibility, and caused little shrinkage in rayon paper.

The interior was reinforced with rayon paper cut to the size of the lacquer layers twice using 3\% funori. We also identified precise locations of broken areas and performed interior facing with rayon paper in the same manner. We also pressed a lead pouch on the surface of the interior while the adhesive was drying to prevent deformation and curling. The rayon paper was cut larger than lacquer layers into a wing shape to facilitate its bonding with reinforcement later(Figure 16).

\subsection{Conservation of the metal}

We used acetone as the solvent for acrylic resin at the recovery site for its vaporization characteristics, but used Xylene in conservation treatment for its characteristic during aging.

We found that the metal part was separated from lacquer part. We also found some wooden pieces and lacquered pieces inside the metal part. This suggests that lacquer was coated on wood and then a metal part was mounted on it. We separated the metal part to perform conservation treatment. The surface of the metal part was entirely covered with chloride and was highly corroded in a very 


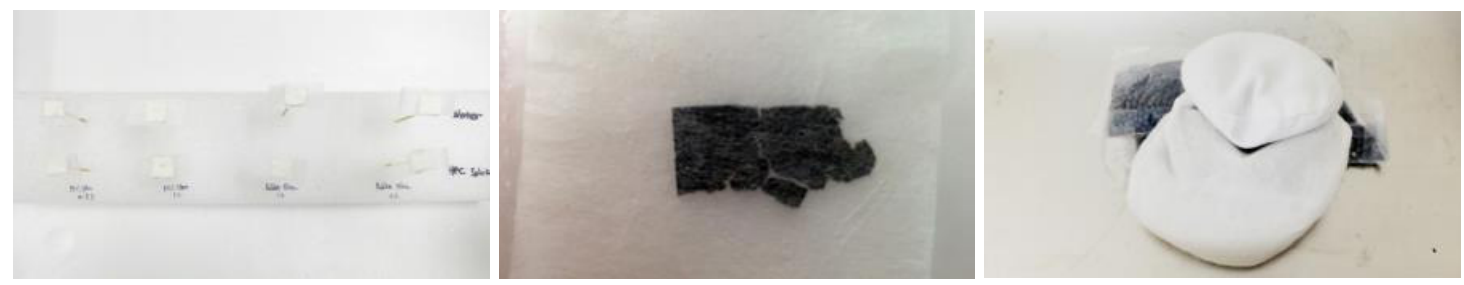

Figure 16. Conservation of hardening (Left: adhesive experiment, Center: reinforcement with rayon paper, Right: drying).
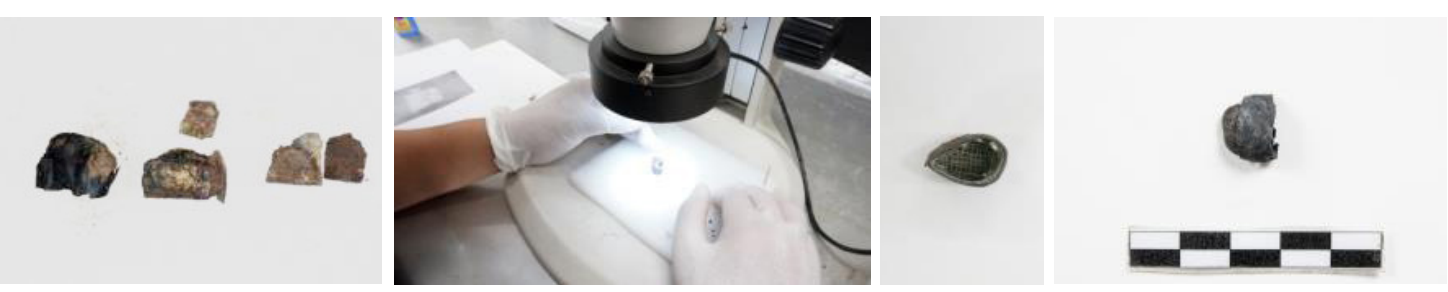

Figure 17. Conservation of metal (Left: condition before treatment, Center: removal of foreign materials, Right: treated metal piece and interior wood piece).
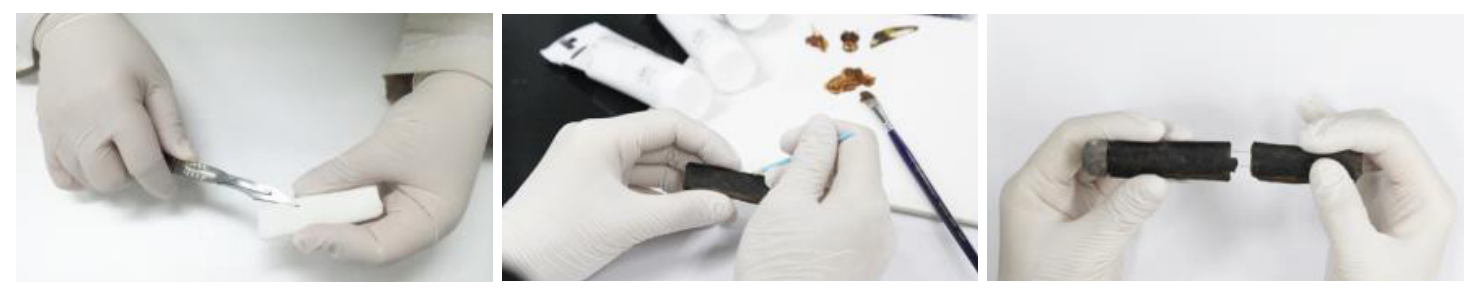

Figure 18. Conservation of supporting (Left: fabricating supporting stand, Center: color blending, Right: restoration of shape).

vulnerable condition. Before applying reinforcement, we removed acrylic resin used when recovering the artifacts and any foreign materials. Then we applied acrylic resin(Paraloid B-72) to the surface for hardening.

After hardening, we performed a joint and restoration work for broken pieces, and reinforced the interior using gauze(Figure 17).

\subsection{Interior reinforcement}

We fabricated the reinforcement for the interior to support the interior cavity and connect the lacquered and metal parts to retain the shape of the lacquered artifacts. We chose easy-to process, lightweight Poly Vinyl Acetate (PVA) as the material for reinforcement. The reinforcement fabricated in the shape of the interior wooden core was

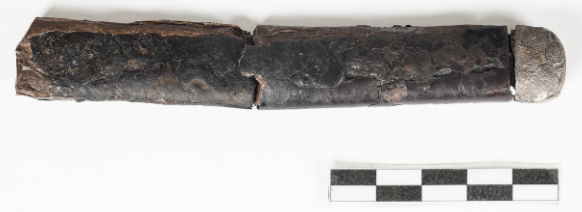

Figure 19. After conservation.

applied using acrylic resin(Paraloid B-72) for the first coating; then rayon paper was applied using funori for second coating. We inserted the reinforcement to keep the shape of the lacquer part, and designed the metal part to be fitted with the section that connects with the lacquer part to facilitate its exhibit in the future. The reinforcement and the lacquerware were attached using funori, and then the rayon 
paper shown through cracks in lacquer layers was painted using acrylic paint for color blending(Figure 18, 19).

\section{CONCLUSION}

Conservation treatment could be successfully applied to the lacquered artifacts excavated from Suchon-ri through quick recovery with maximum preservation.

The lacquered artifacts retained only the lacquer layers with a hollowed interior for the lost interior wood core and the metal part. However, conservation treatment was carried out while retaining the presumably elliptical three-dimensional shape. In particular, the artifacts was treated using materials with reversibility to allow re-examination and retreatment in the future. We also fabricated reinforcement for the interior of the lacquer layers to maintain the present condition.

The examination revealed that the lacquer layers have at least three layers. The component analyses revealed that the metal part was primarily made of $\mathrm{Ag}$, and a small amount of $\mathrm{Cu}$ suggests that it was partially used along with $\mathrm{Ag}$. The wooden pieces found inside the metal part suggests that the silver metal part was fitted with the lacquered wood.

Recently, a large number of excavation reports on lacquered artifacts have been published, including many cases with only the lacquerwares with lost wood parts. However, conservation treatment is often carried out on lacquered artifacts with wood core, and few studies have involved conservation treatment for artifacts with only lacquerwares in three-dimensional shapes. The significant merits of the present study include its examination of conservation treatment strategies for lacquered artifacts with only the three-dimensional shaped lacquerware, and the application of a new conservation technique of filling the hollow interior with reinforcement using clay. However, further research is required to replicate and follow up conservation treatment for lacquered artifacts.

\section{REFERENCES}

Choi, S.C., Kim, S.D., Yi, Y.H., Ko, S.R. and Ham S.W., 2011, Studied on analysis of Joseon lacquer layer. Journal of Conservation Science, 27(4), 371-380. (in Korean with English abstract)

Chungnam Institute of History and Culture, 2013, Ancient tombs in Suchon-ri, Gongju. (in Korean)

Kim, S.C., 2007, Analysis and conservation of lacquer wares from archaeological sites in Korea. Ph. D. dissertation, Chungbuk University, Cheongju. (in Korean with English abstract)

Kim, S.C. and Lee, K.H., 2008, Analysis for species and lacquer ware of wooden object excavated from Sinchang-Dong, Gwangju. Conservation Science in Museum, 9, 95-104. (in Korean with English abstract)

Kim, S.C. and Yi, Y.H., 2004, The conservation treatment for the lacquered sheath excavated from Sinchang-dong, Gwangju. Conservation Science in Museum, 5, 37-41. (in Korean with English abstract)

Lee, K.H. and Han, G.S., 2017, Study on applying techniques of wooden lacquerware artifacts excavated from Imdangdong Site, Gyeongsan, Korea. Journal of Conservation Science, 33(2), 61-73. (in Korean with English abstract)

Lim, J.Y. and Fumio, O., 2018, Analysis of lacquer coating found from Daesungdong No.88 tomb of Gimhae. Journal of Conservation Science, 34(1), 51-57. (in Korean with English abstract)

Yi, Y.H., 2010, A study of the techniques of the ancient Korean lacquer wares. Master's thesis, Kongju National University, Gongju. (in Korean with English abstract)

Zhao, X., Zhang, J., Wang, B and Zada, A., 2015, Biochemical synthesis of $\mathrm{Ag}-\mathrm{AgCl}$ nanoparticles for visible-light-driven photocatalytic removal of colored dyes. Materials $8(5)$, 2043-2053. 